Appeared in: The Third International Conference on Autonomic and Autonomous Systems, ICAS 2007, June 19-25, 2007 - Athens, Greece

\title{
An Investigation of Sharing of Seller Reputation Among Buyers in Agent-Based Markets
}

\author{
Sandhya Beldona, Costas Tsatsoulis \\ Department of Electrical Engineering and Computer Science \\ Information and Telecommunication Technology Center \\ The University of Kansas \\ 2335 Irving Hill Road \\ Lawrence, KS 66045 \\ sbeldona@ittc.ku.edu,tsatsoul@ittc.ku.edu
}

\begin{abstract}
Our work investigates the effects of knowledge sharing among buyer agents in an electronic market, where the shared knowledge is the reputation of seller agents. Each buyer agent independently models a seller after having purchased goods from the seller and, upon request, shares this knowledge with friends. We investigated if having a larger number of friends enables buyers to find good quality low priced sellers in the market more rapidly and also if there is a point of diminishing returns, beyond which increasing the number of friends will not have any positive effects. Our results show that, by sharing information with friends, the buyers are able to identify good sellers earlier. However the marginal gain of having more friends decreases as the number of friends increases.
\end{abstract}

\section{Introduction}

Recent research has developed intelligent agents for ecommerce applications $[1,2,3,5,12,13,14]$. Autonomous agents are suited as mediators in ecommerce due to their personalized, continuous and autonomous nature. The use of agent technologies in the various stages of buying process helps combat information overload, expedites several stages of buying process, and reduces transaction costs [9].

Our work considers domains of tangible goods like ecommerce where there are buyer and seller agents, and where buyers have to repeatedly buy products from sellers. For the same product, the quality and the price may vary across sellers, and, ideally, a buyer would like to obtain the highest quality product at the lowest price possible. The utility for the buyer is defined as: Ubuyer= quality/price. To maximize its utility, the buyer agent can keep trying various sellers until it is satisfied with a particular one and then stick to that seller. However there is no guarantee that the buyer has maximized its utility by choosing this seller to buy from, unless it has bought from all sellers in the marketplace, since there could be other sellers offering higher quality, lower price or both.

A second option could be to explore the market completely until a seller who provides the maximum utility for the buyer is identified. However there is the associated cost to the buyer for trying out sellers selling at higher price, lower quality or both.

These two scenarios assume that the buyer agent can receive quality and cost information only by purchasing goods from the sellers. We propose a market environment where the buyer knows of other agents, trusted friends, who are other buyer agents in the market. These friends can provide information about the reputation of sellers that they have purchased from. As a buyer is collaborating with friends in exploring the market, its own exploration time will be reduced and hence good sellers will be identified faster.

In the rest of this paper we present how a buyer agent creates a model for the reputation of sellers it buys from, and how to combine this information with the seller reputation values provided by friends. Next we discuss how the combined reputation can be used to select the best seller agent. Finally we describe the experiments we ran to study the effect of friends in the utility of purchased goods, and conclude with results from these experiments.

\section{Theoretical Approach}


Our work focuses on markets with a finite set of seller and buyer agents. Agents are considered trustworthy, in that their advice can be taken at face value. Buyers try to maximize their utility, by getting goods and services at the best price and quality.

A buyer uses two criteria in selecting a seller: First, the combined reputation of the seller based on the buyer's own experiences with that seller, and the reputation of that seller as reported by the buyer's friends (i.e., other buyer agents the buyer knows of and trusts). Second, the buyer will evaluate the price which the seller is quoting for the product or service.

In this paper we use the following notation: Subscript represents the agent computing the rating. Superscript represents the agent about whom the rating is being computed. The information in the parenthesis in the superscript is the kind of rating being computed. For example, every time buyer $b$ purchases a product from the seller, it computes a direct trust (di) rating $T_{b}{ }^{\text {s(di) }}$ of the seller $s$ by buyer $b . T_{b}^{s(d i)}$ is computed as $T_{b}^{s(d i)}=$ $\alpha^{*}$ quality. and the value ranges from $[-1,1]$. The rating represents the satisfaction level of the buyer with the seller and is directly proportional to the quality of the product received. A trust rating of -1 is the worst rating implying that the buyer is very unsatisfied with the seller. A rating of 1 implies that the buyer was happy with the seller's quality and service. For sellers that the buyer has no previous experience, the rating is set to 0 .

Each buyer agent records the history of its transactions with the sellers as $\left(s, t, p, p r, T_{b}^{s(d i)}\right)$, where $s$ is the seller's identification, $t$ is the time of interaction, $p$ is the product type, $p r$ is the price, and $T_{b}^{s(d i)}$ is the trust rating of $s$ based on direct interaction.

A buyer $b$ computes the average trust rating $T_{b}^{\text {s(diavg) }}$ of a seller $s$ if it has had at least three direct interactions with that seller, and it only keeps track of its five most recent interactions (we are assuming that a previously bad seller may change and offer better price and quality, and do not want to penalize them for previous bad service):

$$
T_{b}^{s(\text { diavg })}= \begin{cases}\sum_{i=1}^{n} T_{b}^{s(d i)} / n & \text { if } n \geq 3 \\ 0 & \text { if } n<3\end{cases}
$$

If the number of interactions is less than three then the trust rating is set to 0 implying that the buyer $b$ is neutral about the seller s. This ensures that all sellers in the market are given multiple opportunities to prove themselves. When buyer $b$ is queried by friends for the reputation value of a seller $s, b$ sends the value of $T_{b}^{\text {s(diavg) }}$ to its friends.

The buyer $b$ computes the average reputation value $T_{b}^{\text {s(avgrep) }}$ of seller $s$ across its friends as a simple average:

$$
\begin{array}{|l|l|}
\hline T_{b}^{s(\text { avgrep })}=\frac{T_{b 1}^{s(\text { rep })}+T_{b 2}^{s(\text { rep })}+T_{b 3}^{s(\text { rep })}+\ldots \ldots \ldots+T_{b n}^{s(\text { rep })}}{n} & (2 \\
\hline
\end{array}
$$

where $n$ is the number of friends of the buyer returning a non-zero reputation value for $s$, and $T_{b n}{ }^{s(r e p)}$ is the reputation value of seller $s$ returned by friend $b n$.

If a friend returns a value of 0 for the reputation (meaning that it has no reliable information), then this value is not considered in computing the average. We are making two assumptions here: first, friends are trustworthy and do not lie; second, the friends of a buyer rate sellers in a way similar with the buyer agent $b$.

The aggregate trust rating $T_{b}^{s}$ for the seller $s$ by a buyer $b$ is based on its own rating of that seller and the average reputation value of that seller across its friends. There are four distinct scenarios when computing $T_{b}^{s}$

Case1: When the seller is new to the buyer ( $T_{b}{ }^{\text {s(diavg) }}$ $=0$ ) and to its friends $\left(T_{b}^{\text {s(avgrep })}=0\right)$. Then $T_{b}{ }^{s}=0$.

Case 2: When the seller is new to its friends $\left(T_{b}^{\text {s(avgrep })}\right.$ $=0$ ) but the buyer has previously purchased from seller $\left(T_{b}^{\text {s(diavg) }} \neq 0\right)$. Then $T_{b}^{s}=T_{b}^{\text {s(diavg) }}$.

Case 3: When the seller is new to the buyer $\left(T_{b}^{\text {s(diavg) }}\right.$ $=0$ ) but at least one of its friends has purchased from the seller in the past $\left(T_{b}^{\text {s(avgrep) }} \neq 0\right)$. Then $T_{b}^{s}=T_{b}^{\text {s(avgrep) }}$.

Case4: When the seller is not new to the buyer or its friends $\left(T_{b}^{\text {s(diavg) }} \neq 0\right)$ and $\left(T_{b}^{\text {s(avgrep })} \neq 0\right)$. Then

$$
\begin{array}{l|l}
T_{b}^{s}=\frac{T_{b}^{s(d i)}+T_{b}^{s(\text { (avgrep })}}{2} & (3 \\
\hline
\end{array}
$$

In other words, a buyer $b$ fully trusts its friends, and considers their advice of equal importance to its own personal experiences. Clearly, this can be modified to give more or less influence to the advice by the buyer's friends, depending on the level of trust between the buyer and its friends.

A seller $s$ is considered unexplored by buyer $b$ if the aggregate trust rating $T_{b}^{s}$ is zero. While buying goods and services the buyers explore the market place, and, if there are unexplored sellers, one of them is chosen randomly. The goal is to completely explore the market place of finite sellers, while giving all sellers the opportunity to provide the product. This approach is different from the ones where a buyer attempts to identify qualified sellers as quickly as possible (or, at least, in a predetermined period of time), as, for example, in Kasbah [1]. In our scenario it is more beneficial for the buyer to form a complete picture of the market, since it intends to be in it for long periods of time.

After some transactions, all sellers will be explored by the buyer either by directly purchasing from them, or by receiving their reputation by its friends. The aggregate 
trust rating for all the sellers that the buyer is considering is computed and this information is used to choose a seller, together with the price at which it is offering the product or service. Sellers are ranked on reputation in decreasing order. If there are two or more sellers of similar reputation then they are ranked on the prices in increasing order. So a seller who has high reputation and is quoting the lowest price for the product is chosen.

\section{Related Work}

Our approach to modeling the sellers and sharing the reputation of the sellers with friends is similar in part to the models of trust developed in Fire [6] and Regret [11]. These models include other components, such as certified reputation in Fire or social reputation in Regret. Our model also differs from how the direct rating and the reputation value returned by friends is combined.

Zacharia et.al [14] consider a knowledge marketplace where the buyers are users with questions and sellers are the users that can potentially answer these questions. A centralized reputation mechanism as used in the Sporas system [14] is used to update the seller's reputation value based on its most recent transaction. Our model is different as it considers a decentralized marketplace in which there is no central place from which the reputation of the seller can be ascertained. The performance of a seller is estimated based on direct interaction with the seller and on the reputation of the seller as ascertained from trustworthy friends.

Regan et. al. [10] also have a decentralized model of reputation of sellers, and the buyers share reputation of the sellers with each other. Unlike our model, a buyer requests information only when it has no previous experience with the seller. Also they model reputation of friends (whom they refer to as advisors) to account for the subjectivity of rating sellers and of untrustworthy advisors. In our work we are assuming trustworthy friends, and focus on the question of the effect such friends have on the eventual utility of transactions.

Goldman et. al. [5] looked at scenarios where electronic sellers are faced with multiple purchase orders coming from anonymous buyers, and which the sellers need to fulfill with limited stocks. They considered different strategies maximizing the gain for the sellers and counter strategies for buyers. They assumed a market where a finite set of buyers interact repeatedly with a finite set of sellers, and where the buyers submit their purchase orders sequentially and the sellers cannot fulfill all the requests on time. In this environment buyers try to choose sellers who most likely will fulfill their order, and strategy is based only on the service provided by the sellers in the previous encounter only. This can be thought of as trust based on a single direct interaction. We consider the previous history of the seller with the buyer as well as the reputation of this seller with the buyer's friends.

In Kasbah [1] buyer and seller agents autonomously negotiate with each other and try to make the best deal on behalf of their user. The goal for the buyer agent is to find a seller who is offering the product at lowest price within a time limit, and quality is not considered when comparing sellers. Seller agents try to sell their product at the highest price they can sell within a time limit and they reduce the price of their product with the passage of time. The seller's previous history with the buyer or the reputation of the seller is not considered. In our case the seller offers the product at fixed rate and the buyers want to pick a seller who offers a high quality product at low price with no time constraints. We also consider the history of transactions between a seller and a buyer and its friends.

\section{Experiments and Results}

For our experiments we developed a simulation of an electronic market; Figure 1 provides a simplified overview of the market's architecture. The simulation consists of a Matchmaker [7], buyer agents and seller agents, which communicate via KQML messages [4].

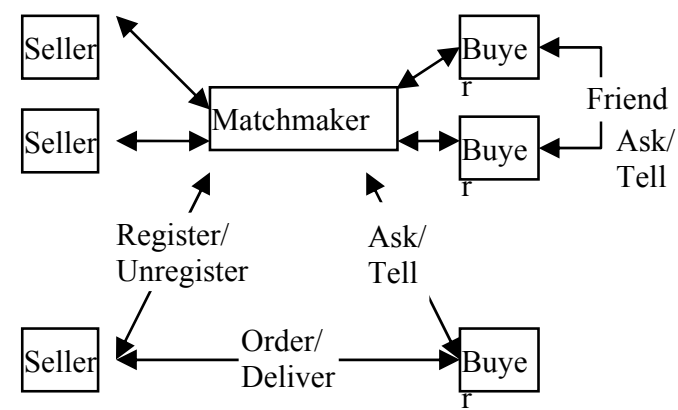

Figure 1. Market Simulation comprising of matchmaker, buyer agent with friend buyer agents and seller agents.

For simplicity, and without loss of generality, in our simulation each seller agent can provide only a single item at a fixed quality and price ranges. Quality and price can be one of LOW, MEDIUM, and HIGH. Each one of these categories represents an actual numerical interval. When a seller provides goods of, say, MEDIUM quality, the actual numerical quality value will be a random number generated from the numerical interval for the category MEDIUM. The same happens for all quality and price categories. This way, while the general quality 
and price of good do not change semantically, they can change in absolute numerical terms.

The quality and price categories with their corresponding intervals are:

Price:

LOW: $[10,16]$

MEDIUM: $[14,20]$

HIGH: $[18,24]$

Quality:

LOW: $[-1.0,-0.7]$

MEDIUM: $[0.3,0.6]$

HIGH: [0.7, 1.0]

Each simulation of the market consists of the following steps.

1)The Matchmaker comes online

2) Sellers enter the market by registering their products types and price categories with the Matchmaker.

3) Buyers enter the market. Each buyer has a predefined set of friends. Each buyer after entering the market waits for some time to let friends join the market.

4) During each iteration, a buyer sends an ask message to the Matchmaker requesting sellers that can provide a certain item or service. The Matchmaker responds by sending a tell message with the list of sellers and the price within the price category of each (as discussed, one of LOW, MEDIUM or HIGH). Next, the buyer sends an ask message to each of its friends requesting reputation information for the sellers on the list. After the information has been received or after waiting for a certain amount of time, the buyer computes each seller's reputation as described in section "Theoretical Approach," and selects the best seller based on the advertised price category and the actual reputation value. Note that the only way for a buyer to experience first hand the quality of a seller is to purchase from it. The quality in the delivered goods is then used to update the seller's history and to modify the buyer's trust in that seller.

5) This process of buying goods continues for any number of iterations.

The goal of our experiments was to study how the number of trustworthy friends in a market can help a buyer identify the best sellers. In the first experiment we fixed a market of ten sellers with price and quality properties as shown in Table1. By predefining the number and quality of sellers we could easily identify the best seller at any iteration. In the second experiment we generated random seller networks where the number of sellers was created randomly, as were the price and quality categories of each seller agent.

Both experiments started with a single buyer agent with no friends, and we ran a number of experiments incrementing the number of friends by one for each experiment. As described, a buyer keeps purchasing from Table 1. Seller properties for fixed seller network for first experiment

\begin{tabular}{|l|l|l|}
\hline $\begin{array}{l}\text { Seller } \\
\text { Identity }\end{array}$ & Price & Quality \\
\hline Seller1 & LOW & LOW \\
\hline Seller2 & LOW & HIGH \\
\hline Seller3 & LOW & $\begin{array}{l}\text { MEDIU } \\
\text { M }\end{array}$ \\
\hline Seller4 & $\begin{array}{l}\text { MEDIU } \\
\text { M }\end{array}$ & LOW \\
\hline Seller5 & $\begin{array}{l}\text { MEDIU } \\
\text { M }\end{array}$ & HIGH \\
\hline Seller6 & $\begin{array}{l}\text { MEDIU } \\
\text { M }\end{array}$ & $\begin{array}{l}\text { MEDIU } \\
\text { M }\end{array}$ \\
\hline Seller7 & HIGH & LOW \\
\hline Seller8 & HIGH & $\begin{array}{l}\text { MEDIU } \\
\text { M }\end{array}$ \\
\hline Seller9 & HIGH & HIGH \\
\hline Seller10 & LOW & HIGH \\
\hline
\end{tabular}

sellers of unknown reputation to explore the market completely, and considers the market explored when the aggregate trust ratings for all the sellers in the market is non zero. After the exploration phase is complete, the buyer starts choosing a seller with high reputation and who is offering the product at the lowest price. We identify this point as a convergence point, and tabulate such points to identify how quickly a buyer reaches them.

Figures 2 and 3 show some of the results of running our experiments on the initial network of the ten fixed sellers. Figure 2 shows the iterations of the system (i.e. buying instances) versus the trust rating assigned to the chosen seller for that iteration based on the quality of the service and product provided. Figure 3 shows the iterations of the system (i.e. buying instances) versus the price at which the product was purchased for that iteration. 


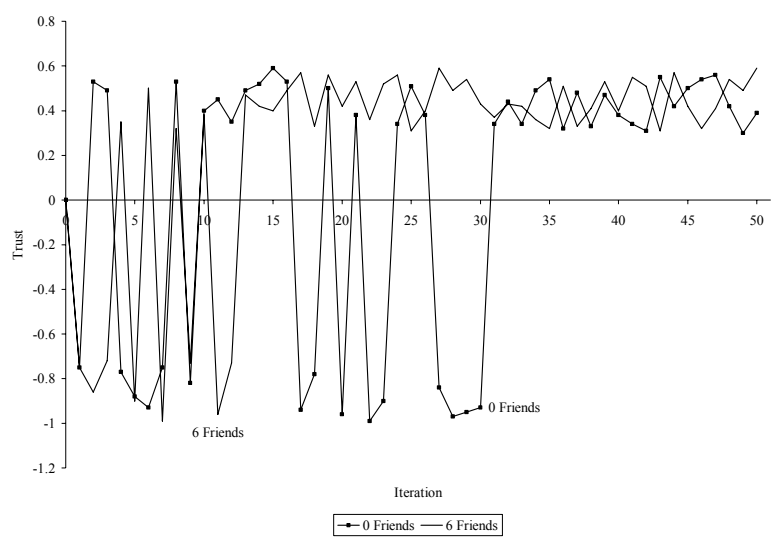

Figure 2. The trust of a selected service/goods provider (seller) vs. buying instance (iteration). Buyer with 6 friends is able to identify and start interacting with sellers who get higher trust rating (high quality low priced) earlier than a buyer with 0 friends.

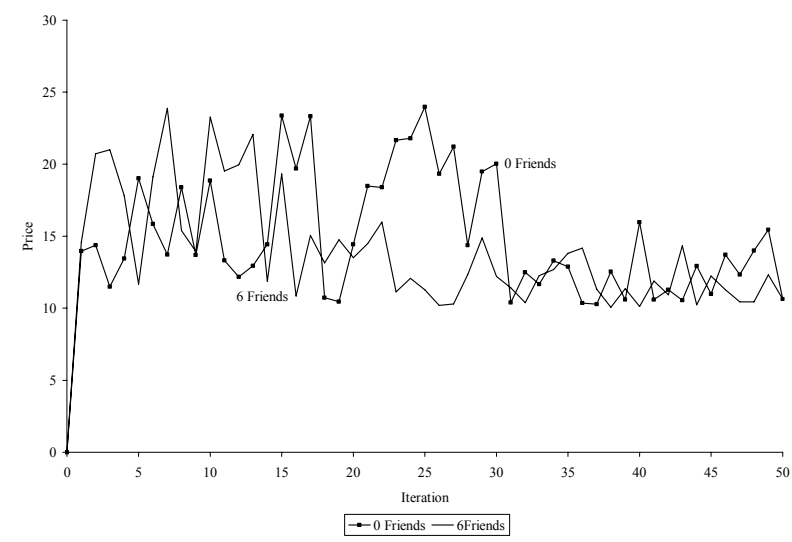

Figure 3. The price of a purchased service/good vs. buying instance (iteration). After buyers converge to the best seller, the price goes down.

Initially, during the market exploration phase, sellers are chosen randomly from the unexplored list of sellers. During this phase we expected the price and trust ratings to vary randomly. After the exploration phase is complete, we expect the buyers to consistently pick high quality low priced sellers from the available sellers in the market. The results conformed to our expectations as is evident from figures 2 and 3. It is also clear that a buyer with friends explores the market faster than a buyer without any friends. There was no substantial difference, though, for convergence for buyers with three friends or more. This is shown best in figure 4 which plots the average converging points (iterations) versus the number of friends for the seller configuration described in Table 1 .

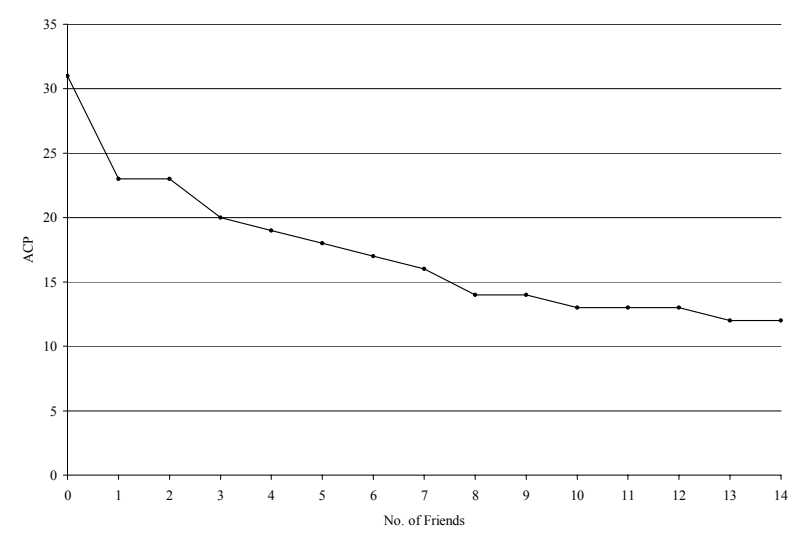

Figure 4. Average convergence point (ACP) vs. the number of friends. As the number of friends increases, the ACP decreases. However the marginal gain by adding more friends decreases as the number of friends increases.

From figure 4 it is evident that adding more friends allows buyers to converge more quickly on acceptable sellers, however, the marginal gain from adding new friends decreases as the number of friends increases.

In the second set of experiments we randomly generated the configuration of the seller network as well as the quality and price of the goods each seller offered. We defined the seller network quality $(S N Q)$ as :

$$
\begin{array}{|l|l|}
\hline S N Q=\sum_{i=1}^{n} \frac{\text { Quality }}{\text { Price }} & (4 \\
\hline
\end{array}
$$

where $n$ is the number of sellers in the market. The $S N Q$ value is a metric of how easy it would be for a buyer to find high quality low price goods. If $S N Q$ is high it implies that there are a lot of high quality low price sellers, while a low value implies the opposite.

We ran the same type of experiments as before, and examined how the quality of the seller network affects the average convergence points for varying sizes of friend network. Figure 5 shows the average convergence points versus seller network quality. 


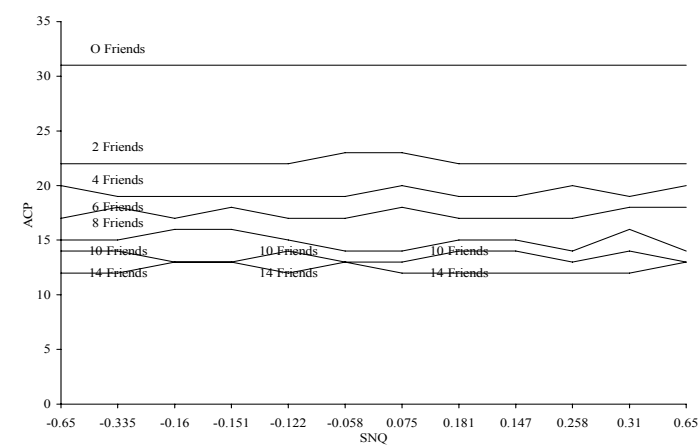

Figure 5. ACP versus SNQ. Irrespective of the seller network quality, the ACP is nearly the same for a given friend network size.

As can be seen from figure 5 the seller network quality does not affect the convergence points. Initially, buyers are not aware of the quality of sellers in the market. Hence they always collaborate and explore the market to identify acceptable sellers. Clearly, though, the number of friends affects the average convergence point. Finally, we studied how the number of sellers would affect the convergence.

Figure 6 shows the results of the experiments where we varied the number of sellers in the network, and, as before, the quality and price categories were established randomly. For the same number of friends, as the number of sellers is increased, it takes longer to identify acceptable sellers. The marginal gain in having more friends is reduced as the number of friends is increased. In general, when there are at least four sellers in the market, the marginal gain is the highest when going from zero to one friend.

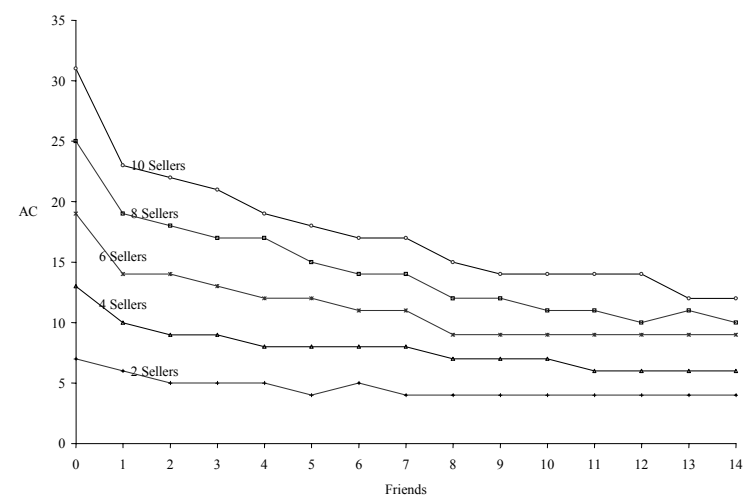

Figure 6. ACP vs. number of friends. A few friends have a dramatic impact as to converging to the best seller. Of course, more sellers require more friends in order to reach convergence.

\section{Conclusions and Future Work}

In a decentralized electronic market place for tangible goods, a buyer agent needs to select a seller before engaging in a transaction with the seller. We have presented a strategy for buyers to model the seller reputation and share this information with friends to identify acceptable sellers quickly. Though our model is similar in part to models of trust and reputation developed in Fire [6] and Regret [11], we investigated if having larger number of friends will consistently enable buyers to find high quality low priced sellers in the market rapidly and also if there is a point of diminishing return, beyond which increasing the number of friends will not have any positive effects. Our results show that by exchanging reputation of sellers with friends, acceptable sellers are identified earlier. However the marginal gain is reduced as the number of friends is increased. In random seller networks, the greatest gain was seen when having a single friend.

\section{Acknowledgements}

This work was supported in part by the National Science Foundation (Grant \#OPP-0122520), the National Aeronautics and Space Administration (grants \#NAG512659 and NAG5-12980), the Kansas Technology Enterprise Corporation, and the University of Kansas.

\section{References}

[1] A. Chavez and P. Maes, "Kasbah: An Agent Marketplace for Buying and Selling Goods," 1st Int. Conf. on the Practical Application of Intelligent Agents and MultiAgent Technology, London, 1996.

[2] A Chavez, D. Dreilinger, R. Guttman. And P. Maes, "A real-Life Experiment in Creating an Agent Marketplace", In Proceedings of the Second International Conference on the Practical Application of Intelligent Agents and MultiAgent Technology, 1997.

[3] R.B Doorenbos, Etzioni, and D. Weld, "A Scalable Comparison-Shopping Agent for the World Wide Web", In Proceedings of the First International Conference on Autonomous Agents, 39-48, 1997.

[4] T. Finn, Y. Labrou, and J. Mayfield, "KQML as an Agent Communication Language," in: J. Bradshaw (Ed.), Software Agents, MIT Press, 1997.

[5] C. Goldman, S. Kraus, and O. Shehory, "Equilibria Strategies: For Selecting Sellers and Satisfying Buyers," Lecture Notes in Artificial Intelligence, Vol. 2182, M. Klusch and F. Zambonelli (Eds.), 166-177, 2001.

[6] D. Huynh, N.R. Jennings, and N.R. Shadbolt, "Developing an Integrated Trust and Reputation Model for Open MultiAgent Systems," 7th Int. Workshop on Trust in Agent Societies, 65-74, 2004. 
Appeared in: The Third International Conference on Autonomic and Autonomous Systems, ICAS 2007, June 19-25, 2007 - Athens, Greece

[7] D. Kuokka and L. Harada, "Supporting Information Retrieval via Matchmaking," Working Notes of the AAAI Spring Symposium on Information Gathering from Heterogeneous, Distributed Environments, 1995.

[8] B. Krulwich, " The bargainfinder agent:comparision price shopping on the Internet", in J. Williams, editor, Bots, and other Internet Beasties, 257-263, Macmillan Computer Publishing, 1996.

[9] P. Maes, R.H.Guttman, A.G.Moukas, “Agents that buy and sell", Communications of the ACM, 42(3), 81-91, 1999.

[10] K. Regan, T. Tran, and R. Cohen, "Sharing Models of Sellers Amongst Buying Agents in Electronic Marketplaces," Decentralized Agent Based and Social Approaches to User Modeling (DASUM), 2005.

[11] J. Sabater and C. Sierra, "REGRET: A Reputation Model for Gregarious Societies," Fifth Int. Conference on Autonomous Agents, Montreal, Canada, 194-195, 2001.

[12] T. Tran and R. Cohen, " Improving User Satisfaction in Agent-Based Electronic Marketplaces by Reputation Modeling and Adjustable Product Quality.", Published in Proceedings of the Third International Joint Conference on Autonomous Agents and Multi Agent Systems (AAMAS-04), Volume 2, 828-835, 2004.

[13] J.M. Vidal and E.H. Durfee, " The impact of Nested Agent Models in an Information Economy", In proceedings of the Second International Conference on Multi-Agent Systems, 377-384, 1996.

[14] G. Zacharia and P. Maes "Trust Management Through Reputation Mechanisms," Artificial Intelligence, 14 (9), 2000. 